\title{
Numerical Modelling of the Tidal Tails of NGC 5466
}

\author{
M. Fellhauer ${ }^{1}$, N. W. Evans ${ }^{1}$, V. Belokurov ${ }^{1}$, M. I. Wilkinson ${ }^{1,2}$ \\ and G. Gilmore ${ }^{1}$ \\ ${ }^{1}$ Institute of Astronomy, University of Cambridge, Madingley Road, Cambridge CB3 0HA, UK \\ ${ }^{2}$ Department of Physics and Astronomy, University of Leicester, University Road, Leicester
} LE1 7RH, UK

\begin{abstract}
The study of sub-structures in the stellar halo of the Milky Way has made a lot of progress in recent years, especially since surveys like the Sloan Digital Sky Survey became available. In this paper we focus on the newly discovered tidal tails of the Galactic globular cluster NGC 5466. By means of numerical simulations we reproduce the tidal tails, which are the longest tails associated with a globular cluster known $\left(>45^{\circ}\right)$ and hereby finding a possible progenitor of NGC 5466 and analyse its stability. We show that perigalactic passages are the dominant process in the slow dissolution of NGC 5466. Furthermore we use the position of the tails to verify the accuracy of the observationally determined proper motion. The proper motion has to be refined only slightly (within their stated error-margin) to match the location of the tidal tails.
\end{abstract}

Keywords. globular cluster: individual (NGC 5466), Galaxy: kinematics and dynamics, Galaxy: halo, methods: n-body simulations

\section{Introduction}

Recently, two different groups (Belokurov et al. 2006; Grillmair \& Johnson 2006) claim to have detected tidal tails of various extents around the globular cluster NGC 5466. This is an old, metal-poor $([\mathrm{Fe} / \mathrm{H}]=-2.22)$ cluster, lying at Galactic coordinates $l=42.15$, $b=73.59$. In Belokurov et al. (2006), the observed tails of NGC 5466 are not as long as those of Pal 5 , stretching about $2^{\circ}$ or $500 \mathrm{pc}$ in either direction. Grillmair \& Johnson (2006) reported afterwards that they found evidence for a much larger extension of the tidal tails of NGC 5466. They claimed that the leading arm extends over $\sim 30$ degrees and the trailing arm extends at least 15 degrees, before it leaves the area covered by SDSS. This finding makes the tails of NGC 5466 even longer, but much fainter, than the tails of Pal 5. The aim of our paper is to confront these claims with theoretical expectation, as well as to study the survival of the cluster and the mechanisms of its mass-loss.

\section{Setup}

We use the particle-mesh code SupERBox (Fellhauer et al. 2000) to carry out our simulations. The Milky Way potential is added analytically consisting of a Hernquist bulge, a Miamoto-Nagai disc and a logarithmic halo with parameters described in Fellhauer et al. (2007). The globular cluster NGC 5466 is modelled as a Plummer sphere with Plummer radius of $10 \mathrm{pc}$ and initial mass of $7 \times 10^{4} \mathrm{M}_{\odot}$. 


\section{Results}

All our results can be found in detail in Fellhauer et al. (2007).

By running particle-mesh simulations using a million particles for the cluster we are able to trace its tidal tails in great detail. We find that the location of the near-field tails (around the cluster, as seen by Belokurov et al. 2006) do not conform with the proper motion given in the literature (Dinescu, Girard \& Altena 1999). The proper motion has to be slightly (within the given error-margins) altered to $\mu_{\alpha} \cos \delta=-4.7 \operatorname{mas~yr}^{-1}$ and $\mu_{\delta}=0.42$ mas yr$^{-1}$ to give the model tails the same orientation as the observed ones. We confirm the extend of the tidal tails and their density of about $25 \mathrm{M}_{\odot} \operatorname{deg}^{-2}$ as claimed by Grillmair \& Johnson (2006).

By comparing the mass-loss of the particle-mesh simulation with a direct N-body simulation, we show that only $1 / 3$ of the mass-loss is caused by internal evolution and the major reason for the mass-loss is tidal shocking. We confirm the results of Gnedin, Lee \& Ostriker (1999), that the initial NGC 5466 as well as the present cluster are able to survive for at least a Hubble-time and that the cluster resides in a regime where shocks are important. Calculating the disc-shock time-scale gives 110 Gyr for NGC 5466. In our simulations we found that without a disc present the mass-loss is reduced from 19 per cent down to 3 per cent over $10 \mathrm{Gyr}$ of simulation. But a detailed inspection of the massloss at each pericentre- and disc-passage shows that it is not the actual disc-shock (i.e. the cluster travelling through the disc) but rather the general pericentre passage which is responsible for the bulk of the lost mass.

\section{Conclusions}

We have presented numerical simulations of the formation and evolution of the tidal tails of the globular cluster NGC 5466. We used direct N-body codes to argue that the evolution of the cluster is dominated by external effects rather than internal relaxation, and then grid-based codes to trace the faint tidal tails. This novel, hybrid approach is well-suited to map out the detailed morphology of the low-density tails of NGC 5466 .

Naively, we might expect that a low mass cluster with observed and very lengthy tails on a disc crossing orbit would not be able to survive for too much longer. However, simulations by Dehnen et al. (2004) have already shown that the disrupting globular cluster Pal 5 has survived for at least many Gyr in a tidally-dominated and out-ofequilibrium state, although Pal 5 probably will be destroyed at the next disc crossing. Here, we have demonstrated that a progenitor cluster of NGC 5466, which is quite similar to the present cluster, could survive substantially longer, for at least a few Hubble times, with its extensive but tenuous tidal tails gradually wrapping around the whole Galaxy.

\section{References}

Belokurov, V., Evans, N. W., Irwin, M. J., Hewett, P. C., \& Wilkinson, M. I. 2006, ApJL 637, L29

Dehnen, W., Odenkirchen, M., Grebel, E. K., \& Rix, H.-W. 2004, AJ 127, 2753

Dinescu, D. I., Girard, T. M., \& van Altena, W. F. 1999, AJ 117, 1792

Fellhauer, M., Kroupa, P., Baumgardt, H., Bien, R., Boily, C. M., Spurzem, R., \& Wassmer, N. 2000, New Astron., 5, 305

Fellhauer, M., Evans, N. W., Belokurov, V., Wilkinson, M. I., \& Gilmore, G. 2007, MNRAS 380,749

Gnedin, O. Y., Lee, H. M., \& Ostriker, J. P. 1999, ApJ 522, 935

Grillmair, C. J. \& Johnson, R. 2006, ApJL 639, L17 